\title{
Validation of the Publication of New Names and New Combinations Previously Effectively Published Outside the IJSB
}

\author{
List No. $49 \dagger$
}

The purpose of this announcement is to effect the valid publication of the following new names and new combinations under the procedure described previously [Int. J. Syst. Bacteriol. 27(3):iv, 1977]. Authors and other individuals wishing to have new names and/or combinations included in future lists should send the pertinent reprint or a photocopy thereof to the IJSB (c/o ASM) for confirmation that all of the other requirements for valid publication have been met. It should be noted that the date of valid publication of these new names and combinations is the date of publication of this list, not the date of the original publication of the names and combinations. The authors of the new names and combinations are as given below, and these authors' names will be included in the author index of the present issue and in the volume author index in this issue of the IJSB.

Inclusion of a name on these lists validates the name and thereby makes it available in bacteriological nomenclature. The inclusion of a name on this list is not to be construed as taxonomic acceptance of the taxon to which the name is applied. Indeed, some of these names may, in time, be shown to be synonyms, or the organisms may be transferred to another genus, thus necessitating the creation of a new combination.

\begin{tabular}{|c|c|c|c|c|}
\hline Name & Proposed as: & Author(s) (reference) & Priority $^{a}$ & Nomenclatural type ${ }^{b}$ \\
\hline Corynebacterium propinquum & New species & Riegel et al. (6) & 2 & Strain B 77159 (= CIP 103792) \\
\hline Dolosigranulum & New genus & Aguirre et al. (1) & 4 & Dolosigranulum pigrum \\
\hline Dolosigranulum pigrum & New species & Aguirre et al. (1) & 4 & Strain R91/1468 (= NCFB 2975) \\
\hline Desulfobacterium cetonicum & New species & Galushko and Rozanova (4) & 3 & Strain $480(=$ VKM B-1975 = DSM 7267) \\
\hline Desulfomicrobium & New genus & Rozanova et al. (7) & 3 & Desulfomicrobium baculatus \\
\hline Desulfomicrobium apsheronum & New species & Rozanova et al. (7) & 3 & Strain 1105 (= VKM B-1804 = DSM 5918) \\
\hline $\begin{array}{l}\text { Desulfomicrobium baculatus } \\
\text { (basonym Desulfovibrio } \\
\text { baculatus) }\end{array}$ & New combination & Rozanova et al. (7) & 3 & Strain $x(=$ VKM B-1378 = DSM 4028) \\
\hline Helicobacter canis & New species & Stanley et al. (8) & 2 & NCTC $12739=$ ATCC 51401 \\
\hline Rarobacter incanus & New species & Yamamoto et al. (9) & 6 & Strain YLM-32 (= JCM 6350) \\
\hline Tetragenococcus & New genus & Collins et al. (3) & 1 & Tetragenococcus halophilus \\
\hline $\begin{array}{l}\text { Tetragenococcus halophila } \\
\text { (basonym Pediococcus } \\
\text { halophilus) }\end{array}$ & New combination & Collins et al. (3) & 1 & ATCC $33315=$ NCDO $1635=$ DSM 20339 \\
\hline Thermoanaerobium acetigenum & New species & Nielsen et al. (5) & 5 & Strain X6B (= DSM 7040) \\
\hline Weissella & New genus & Collins et al. (2) & 4 & Weisella viridescens \\
\hline $\begin{array}{l}\text { Weissella confusa (basonym } \\
\text { Lactobacillus confusa) }\end{array}$ & New combination & Collins et al. (2) & 4 & ATCC 10881 \\
\hline $\begin{array}{l}\text { Weissella halotolerans (basonym } \\
\text { Lactobacillus halotolerans) }\end{array}$ & New combination & Collins et al. (2) & 4 & ATCC $35410=$ DSM 20190 \\
\hline Weissella hellenica & New species & Collins et al. (2) & 4 & Strain LV346 (= NCFB 2973) \\
\hline $\begin{array}{l}\text { Weissella kandleri (basonym } \\
\text { Lactobacillus kandleri) }\end{array}$ & New combination & Collins et al. (2) & 4 & DSM $20593=$ ATCC 51149 \\
\hline $\begin{array}{l}\text { Weissella minor (basonym } \\
\text { Lactobacillus minor) }\end{array}$ & New combination & Collins et al. (2) & 4 & ATCC $35412=$ DSM 20014 \\
\hline $\begin{array}{l}\text { Weissella paramesenteroides } \\
\text { (basonym Leuconostoc } \\
\text { paramesenteroides) }\end{array}$ & New combination & Collins et al. (2) & 4 & ATCC $33313=$ NCDO $803=$ DSM 20288 \\
\hline $\begin{array}{l}\text { Weissella viridescens (basonym } \\
\text { Lactobacillus viridescens) }\end{array}$ & New combination & Collins et al. (2) & 4 & ATCC $12706=$ DSM $20410=$ NCDO 1655 \\
\hline
\end{tabular}

† Lists 1 through 48 were published in the Int. J. Syst. Bacteriol. 27:306, 1977; 29:79, 436, 1979; 30:601, 676, 1980; 31:215, 382, 1981; 32:266, 384, 1982; 33:438, 672, 896,$1983 ; \mathbf{3 4}: 91,270,355,503,1984 ; \mathbf{3 5}: 223,375,535,1985 ; \mathbf{3 6}: 354,489,573,1986 ; \mathbf{3 7}: 179,1987 ; \mathbf{3 8}: 136,220,328,449,1988 ; \mathbf{3 9}: 93,205,371,495,1989 ; \mathbf{4 0}: 105,212$,

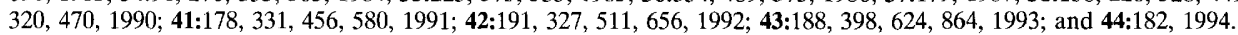

${ }^{a}$ Priority number assigned according to the date the documentation and request for validation are received.

' Abbreviations: ATCC, American Type Culture Collection, Rockville, Md.; CIP, Collection of the Institut Pasteur, Paris, France; DSM, Deutsche Sammlung von Mikroorganismen und Zellkulturen GmbH, Braunschweig, Federal Republic of Germany; JCM, Japan Collection of Microorganisms, Riken, Saitama, Japan; NCDO (National Collection of Dairy Organisms), AFRC Institute of Food Research, National Collection of Food Bacteria, Shinfield, Reading, Berkshire, United Kingdom; NCFB, National Collection of Food Bacteria, AFRC Institute of Food Research, Reading, Berkshire, United Kingdom; NCTC, National Collection of Type Cultures, London, United Kingdom; VKM, All-Union Collection of Microorganisms, Russian Academy of Sciences, Institute of Biochemistry and Physiology of Microorganisms, Moscow Region, Russia. 


\section{REFERENCES}

1. Aguirre, M., D. Morrison, B. D. Cookson, F. W. Gay, and M. D. Collins. 1993. Phenotypic and phylogenetic characterization of some Gemella-like organisms from human infections: description of Dolosigranulum pigrum gen. nov., sp. nov. J. Appl. Bacteriol. 75:608-612.

2. Collins, M. D., J. Samelis, J. Metaxopoulos, and S. Wallbanks. 1993. Taxonomic studies on some Leuconostoc-like organisms from fermented sausages: description of a new genus Weissella for the Leuconostoc paramesenteroides group of species. J. Appl. Bacteriol. 75:595-603.

3. Collins, M. D., A. M. Williams, and S. Wallbanks. 1990. The phylogeny of Aerococcus and Pediococcus as determined by $16 \mathrm{~S}$ rRNA sequence analysis: description of Tetragenococcus gen. nov. FEMS Microbiol. Lett. 70:255-262.

4. Galushko, A. S., and E. P. Rozanova. 1991. Desulfobacterium cetonicum sp. nov.: a sulfate-reducing bacterium which oxidizes fatty acids and ketones. Mikrobiologiya 60:102-107.
5. Nielsen, P., I. M. Mathrani, and B. K. Ahring. 1993. Thermoanaerobium acetigenum spec. nov., a new anaerobic, extremely thermophilic, xylanolytic non-spore forming bacterium isolated from an Icelandic hot spring. Arch. Microbiol. 159:460-464.

6. Riegel, P., D. de Briel, G. Prevost, F. Jehl, and H. Monteil. 1993. Proposal of Corynebacterium propinquum sp. nov. for Corynebacterium group ANF-3 strains. FEMS Microbiol. Lett. 113:229-234.

7. Rozanova, E. P., T. N. Nazina, and A. S. Galushko. 1988. Isolation of a new genus of sulfate-reducing bacteria and description of a new species of this genus, Desulfomicrobium apsheronum gen. nov., sp. nov. Mikrobiologiya 57:634-641.

8. Stanley, J., D. Linton, A. P. Burnens, F. E. Dewhirst, R. J. Owens, A. Porter, S. L. W. On, and M. Costas. 1993. Helicobacter canis sp. nov., a new species from dogs: an integrated study of phenotype and genotype. J. Gen. Microbiol. 139:2495-2504.

9. Yamamoto, N., S. Sato, H. Miki, Y. K. Park, and M. Tadenuma. 1993. Taxonomic studies on yeast-lysing bacteria, and a new species Rarobacter incanus. J. Gen. Appl. Microbiol. 39:261-272. 